\title{
Publisher Correction: A circumbinary protoplanetary disk in a polar configuration
}

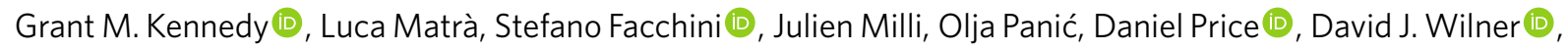
Mark C. Wyatt and Ben M. Yelverton

Correction to: Nature Astronomy https://doi.org/10.1038/s41550-018-0667-x, published online 14 January 2019.

In the version of this Letter originally published, the Author Contributions section was mistakenly omitted, and should have read: "G.M.K. conceived the project, analysed the data, carried out the modelling and wrote the manuscript. L.M. contributed gas calculations and provided advice on self-calibration. B.M.Y. set up and ran the $n$-body simulations. D.P. provided advice on running the smoothed particle hydrodynamics simulations. All co-authors provided input on the manuscript." In addition, in the fourth paragraph, due to a typographical error the value of the ascending node was incorrect as $289 \pm 1^{\circ}$ and should have been $337.6 \pm 2.4^{\circ}$. These errors have now been corrected. 